\title{
Centro de informação sobre medicamentos: contribuição para o uso racional de fármacos
}

\author{
Drug information centre: \\ contribution for rational use of drugs
}

\author{
Cléber Domingos Cunha da Silva 1 \\ Helena Lutéscia Luna Coel ho 1 \\ Paulo Sérgio Dourado Arrais 1 \\ Francisco Romero Cabral 1
}

\footnotetext{
1 Grupo de Prevenção ao Uso Indevido de Medicamentos, Centro de Informação sobre Medicamentos, Universidade Federal do Ceará. Rua Capitão Francisco Pedro 1210 Fortaleza, CE 60431-327, Brasil.
}

Abstract This paper describes the first year experience of a Drug Information Center di rected to the community, and managed by pharmacysts and pharmacy students from the Department of Pharmacy-UFC, in Fortal eza, Brazil. This pioneer experience recieves technical assi stance from the “Centro Regionali di Informazione sul Farmaco-CRIF (Mario Negri Institute Milan-Italy). Those who consult us are registered on a codified formullary whileindividual information, questions and prepared answers are introduced on a database. From December 1994 to November 1995, 246 persons tel ephoned CIM-UFC for drug information of which 39\% were medicine users, $32 \%$ pharmacysts, $17 \%$ pati ents relatives, $4,5 \%$ health students, $4 \%$ doctors, $0,8 \%$ nurses and $2,8 \%$ others. The princi pal questi ons were related to Adverse Reactions (28\%) Indications (28\%) and Efficacy (18\%). The ATC 2nd level groups most referred to were vitamins, analgesi cs and antiinflammatory agents. The most frequent problems justifying drug use were classified as: Endocrine, nutritional and metabolic diseases and immunity disorders; Symptoms, signs and ill defined conditions; Infecti ous and parasitic di seases. Requests are increasing regularly, responding to the mass media campaigns related to drugs and active information is provided by bulletins.

Key words Drug Information Centre; Pharmacoepi demiology; Advice for the Patient

Resumo Esteartigo descreve o primei ro ano de atividades do Centro de Informação sobre Medicamentos da Universi dade Federal do Ceará, em Fortaleza, Brasil. O CIM faz parte do GPU IM (Grupo de Prevenção ao Uso Indevido de Medicamentos, do Departamento de Farmácia), sendo operacional izado por farmacêuti cos especializados. O serviço atende à comuni dade em geral e aos profissionais da saúde em parti cular. De dezembro de 1994 a novembro de 1995, 246 pessoas tel efonaram ao CIM-UFC, das quais 39\% eram usuários de medicamentos, 32\% farmacêuticos, $17 \%$ parentes e/ou ami gos dos usuários, 4,5\% estudantes de Farmácia e/ou de Medicina, 4\% mé dicos, $0,8 \%$ enfermei ras e 2,8\% outros. Os temas mais abordados foram Reações Adversas (28\%) Indicações (28\%) e Efi cáci a (18\%), sen do os grupos de fármacos mai s freqüentes: Vitaminas, Anal gési cos e Agentes Anti inflamatórios. O número de sol i citações vem aumentando regularmente, variando com a divulgação do servi ço através da mídia local e de publicações específicas. Nesse período, o Centro vem esboçando o perfil de um serviço que pode preencher lacunas importantes não só do Sistema de Saúde, como também da formação e prática de médicos efarmacêuticos. Além disso, o Centro está se firmando como uma fonte segura de informações auxiliares nas deci sões das pessoas lei gas no que diz respei to à automedi cação.

Palavras-chave CIMs; Informação para Pacientes; Farmacoepidemiologia 


\section{Introdução}

A disponibilidade de fontes de informação técnico-científica sobre medicamentos e sua utilização apropriada, as quais sejam confiáveis, atualizadas e independentes, são requisitos indispensáveis para garantir o uso racional de medicamentos. Os Centros de Informação sobre Medicamentos (CIMs), definidos como “unidades operacionais que proporcionam informações técni co-ci entíficas sobre medicamentos de modo objetivo e oportuno, constituem uma estratégi a para atender as necessi dades particulares de informação" (CIM, 1995). O primeiro centro de informação sobre medicamentos foi criado em 1962 no Centro Médico da Universidade de Kentucky - Estados Unidos (Amerson, 1996), e hoje já existem centenas de centros em todo o mundo, dentro e fora de unidades hospitalares (Bonati \& Tognoni, 1995; CIM , 1995; Joshi, 1995; Anon, 1995).

O Centro de Informação sobre Medicamentos da Universidade Federal do Ceará (CIM/ UFC) iniciou formalmente a sua atividade em dezembro de 1994, com o objetivo de auxiliar profissionais da saúde e a comunidade em geral em suas dúvidas nesta área. Os operadores do CIM/UFC são farmacêuticos treinados em avaliação de documentação biomédica e informação sobre medicamentos e, quando necessário, contam com a colaboração de um corpo de consultores médicos, bem como de profissionais de outras áreas.

As fontes de informação são aquelas clássicas para um serviço dessa natureza: textos fundamentais de farmacologia básica e clínica, livros sobre reações adversas e avaliação de medicamentos, guias terapêuticos, farmacopéias, textos de fisiopatologia e clínica médica, os mais importantes boletins sobre fármacos, bases de dados (Micromedex, LILACS) (Mosdell \& Malone, 1996). Através da biblioteca do Centro de Ciências da Saúde (CCS), o CIM/UFC tem acesso a Medline e a periódicos da área biomédica. Além disso, o serviço mantém estreito contato com centros semelhantes do Brasil e de outros países.

A constituição e o funcionamento do CIM/ UFC são produtos de uma colaboração realizada, por dois anos, com o CRIF (Centro Regional de Informação sobre Fármacos, do Instituto Mario Negri, Milão-Itália), com o apoio financeiro da SIFO (Sociedade Italiana de Farmácia Hospitalar).

\section{Metodologia}

O CIM/UFC atende à população em geral e aos profissionais de saúde em particular, e a sua atividade prevê:

a) Informação passiva: inclui o atendimento aos solicitantes (em geral por via telefônica), bem como a análise das perguntas, pesquisa em fontes de informação, avaliação das informações obtidas e elaboração da resposta, que pode ser fornecida de forma oral ou escrita, de imediato ou no prazo de dois dias, conforme a necessidade e possibilidade. Todas as informações referentes à solicitação, inclusive a resposta, são registradas em banco de dados, usando-se para isso uma ficha padrão que permite a identificação do usuário do medicamento, do(s) problema(s), do(s) medicamento(s), da natureza da solicitação e da resposta, bem como das fontes de informação empregadas na elaboração da mesma.

b) Informação ativa: elaboração de boletins e artigos sobre assuntos relativos a medicamentos; participação em congressos científicos; ocupação de espaço na mídia, seja por iniciativa desta ou do CIM/UFC; colaboração no programa semanal de rádio: Falando de Medicamentos, produzido pelo GPUIM.

c) Pesquisa: desenvolvimento de estudos de utilização de medicamentos e outros tipos de investigações voltadas para problemas detectados através de perguntas endereçadas ao CIM/UFC.

d) Intervenção: planejamento e execução de intervenções sobre problemas detectados.

\section{Alguns resultados}

Em seu primeiro ano de atividade (dezembro de 1994 a novembro de 1995), o CIM/UFC atendeu a 246 solicitantes: destes, 38,6\% ( $n=$ 95) eram os próprios usuários do(s) medicamento(s); $17,1 \%(n=42)$ eram familiares e/ ou amigos destes; $32,1 \%(n=79)$ profissionais farmacêuticos; 4,5\% ( $n=11)$ estudantes de Farmácia e Medicina; 4,1\% $(n=10)$ médicos; $2,8 \%$ $(n=7)$ outros solicitantes e $0,8 \%(n=2)$ enfermeiros. É interessante observar que o CIM/ UFC foi a primeira fonte de informação à qual se dirigiram 34,6\% dos solicitantes. Dos 296 pedidos de informação: 135 (45,61\%) corresponderam a dúvidas sobre terapêutica (indicação, eficácia, posologia, farmacocinética, composição, tratamento e alternativas terapêuticas); 125 (42,23\%) a problemas com o uso de medicamentos (reações adversas, interações medicamentosas, precauções, toxicidade); 16 (5,40\%) 
casos correspondiam a pedidos de monografia sobre fármacos; 15 (5,07\%) a informações sobre disponibilidade no mercado e cinco $(1,69 \%)$ a outros temas. As principais justificativas para o uso dos medicamentos que motivaram as perguntas (de acordo com a Classificação Internacional das Doenças - CID/10) foram doenças endócrinas, nutricionais e metabólicas, $10,9 \%$ ( $n=27)$; sintomas, sinais e afecções mal-definidas, $10,9 \%(n=27)$ e doenças infecciosas e parasitárias, 9,7\% ( $n=24)$. O perfil das solicitações é completado pela Tabela 1, que documenta os grupos de fármacos que foram objeto de solicitações.

\section{Discussão}

O CIM/UFC está situado em um país no qual a política de medicamentos é ainda muito débil e caracterizada pelas mais diversas formas de resistência ao uso adequado de medicamentos. A sua localização em um ambiente universitário favorece uma forte interação com outros grupos de pesquisa e com atividades de ensino. A experiência de um ano pode ser considerada satisfatória, levando-se em conta que a divulgação do serviço foi realizada em campanhas muito fragmentadas (por problemas de divulgação e escassez de recursos). A divulgação do trabalho do CIM/UFC em congressos nacionais de farmacêuticos fez com que chegassem ao Centro pedidos de informação de outras cidades do Ceará, bem como (embora esporadicamente) de outros estados brasileiros.

Uma das características mais interessantes do perfil do Centro é a forte presença de perguntas provenientes diretamente de pacientes, usuários de medicamentos. O fato não é surpreendente se considerarmos que, particularmente no Brasil, poucos médicos e farmacêuticos orientam adequadamente seus pacientes a respeito do uso adequado de medicamentos (Lopes et al., 1996). A possibilidade de que grande parte desses usuários do Centro estejam utilizando o serviço para fundamentar meIhor decisões relativas à automedicação é levada em conta no atendimento e é um dos objetivos do CIM/UFC. A prevalência dos farmacêuticos entre os solicitantes do CIM/UFC em relação aos médicos é um fenômeno esperado, seja pela localização do Centro numa Faculdade de Farmácia, seja pela tradicional resistência dos médicos em reconhecerem a própria carência informativa.

É interessante observar que a posição dos fármacos mais citados nas solicitações (Tabela

\section{Tabela 1}

Principais subgrupos de fármacos envolvidos nas solicitações apresentadas ao CIM/UFC* (classificação ATC, 2o nível).

\begin{tabular}{|c|c|c|c|c|c|}
\hline ATC & Subgrupos & $\mathrm{n}$ & Fármacos & $\mathrm{n}$ & $\%$ \\
\hline \multirow[t]{2}{*}{ A11 } & Vitaminas & 28 & Complexo B, incl. combinações & 6 & 21,4 \\
\hline & & & Ácido ascórbico & 5 & 17,8 \\
\hline \multirow[t]{3}{*}{$\mathrm{N} 02$} & Analgésicos & 28 & Dipirona & 9 & 32,1 \\
\hline & & & Ácido acetilsalicílico & 4 & 14,3 \\
\hline & & & Dipirona, comb. excl psicoléptic. & 4 & 14,3 \\
\hline \multirow[t]{2}{*}{ M01 } & Produtos antiinflamatórios e anti-reumáticos & 24 & Paracetamol & 3 & 10,7 \\
\hline & & & Diclofenaco & 11 & 45,8 \\
\hline \multirow[t]{2}{*}{ V03 } & Todos os demais produtos terapêuticos & 22 & Piroxicam & 4 & 16,7 \\
\hline & & & Fitoterápicos & 15 & 68,2 \\
\hline A 16 & e metabolismo & & Laxativos & 3 & 16,6 \\
\hline N 03 & Antiepilépticos & 12 & Fenobarbital & 6 & 50 \\
\hline D10 & Preparações antiacne & 6 & Retinol & 5 & 83 \\
\hline $\mathrm{H} 03$ & Terapia tiroidiana & 5 & Levotiroxina & 4 & 80 \\
\hline
\end{tabular}

\footnotetext{
* Somente estão citados os fármacos com freqüência igual ou superior a 3,0.
} 
1), bem como a das queixas que motivaram o uso (ver resultados), não difere do que se observa nos estudos sobre automedicação realizados no Brasil e em outros países latino-americanos (Arrais et al., no prelo; Durgla, no preIo). É também evidente a predominância de medicamentos de eficácia ou segurança duvidosa, como é o caso dos complexos vitamínicos, dipirona, fitoterápicos, cartilagem de tubarão, retinol e outros (Chetley, 1994). Entre as práticas que merecem intervenção, destacamos: o uso excessivo de dipirona, que é dominante em relação ao paracetamol para o controle da febre e de dores; o emprego de antihistamínicos como estimulantes do apetite; o uso de hormônios sistêmicos para feminilização. A relativa freqüência de pedidos de informação acerca dos fitoterápicos, que refletem a difusão tradicional do uso de plantas medicinais em nosso país, levou o serviço a estabelecer uma colaboração com o setor de fitoterápicos do Departamento de Farmácia da Universidade Federal do Ceará.

\section{Conclusão}

A população brasileira não tem a sua disposição serviços sanitários de qualidade: de um lado, a escassez de recursos disponíveis para a saúde faz com que proliferem as cooperativas médicas; de outro lado, o mercado farmacêuti- co propõe uma oferta caracterizada por um excesso de produtos com um perfil insatisfatório de eficácia e segurança (Barros, 1995; Arrais et al., no prelo). É ainda freqüente a presença em nosso mercado de medicamentos que já foram retirados do mercado internacional, tais como dipirona e diversas associações medicamentosas. O emprego de produtos à base de plantas medicinais parece ser uma tentativa de contornar as dificuldades de acesso aos medicamentos e a uma assistência médica/farmacêutica adequadas. A ausência de iniciativas de formação e informação capazes de assegurar competência e interesse nos prescritores torna a situação ainda mais precária e de risco. “Não é fácil prever qual será o impacto, a médio elongo prazo, de um centro de informação qualificado e independente: não há dúvida que a ne cessi dade de um centro dessa natureza é muito grande, equea decisão de dar prioridade ao desenvolvimento de uma estratégi a informativa arti culada e di versi ficada parece ser um caminho válido, seja para os profissionais de saúde seja para a população." (Da Silva, 1996)

Os Centros de Informação sobre Medicamentos (CIMs) podem atuar como observatórios não somente das práticas, mas sobretudo das culturas dominantes nessa área, podendo assim fornecer subsídios às políticas gerais de medicamentos, bem como a estratégias para a abordagem de problemas localizados (Da Silva et al., no prelo).

\section{Agradecimentos}

À Sra. Lynn Silver (IDEC - Instituto Nacional de Defesa do Consumidor) pela colaboração recebida.

\section{Referências}

AMERSON, A. B., 1996. Introduction to the concept of drug information. In: Drug Information: A Guide for Pharmacists (P. M. Malone, K. W. Mosdell, K. L. Kier \& J. E. Stanovich, eds.), pp. 3-11. Connecticut: Appleton \& Lange.

ANON, 1995. Philippines' Drug Information Centre making a real contribution. Essential Drugs Monitor, 20:4.

ARRAIS, P. S. D.; COELHO, H. L. L.; BATISTA, M. C.; CARVALHO, M. L.; RIGHI, R. E. \& ARNAU, J. M. Um perfil da automedicação no Brasil. Revista de Saúde Pública, (no prelo). 
BARROS, J. A. C., 1995. Propaganda de Medicamentos Atentado à Saúde? São Paulo: Hucitec/Sobravime.

BONATI, M. \& TOGNONI, G., 1995. The complex profile of health information. In: Health Information Centre in Europe (M. Bonati \& G. Tognoni, eds.), pp. 7-10, Bergamo: Ed. International Society of Drug Bulletins (ISDB)/ Istituto di Ricerche Farmacologiche Mario Negri.

CIM (CENTROS DE INFORMACION DE MEDICAMENTOS), 1995. Una Estrategia de Apoyo al uso Racional de Medicamentos. Santo Domingo: OMS.

CIM (CENTROS DE INFORMACION DE MEDICAMENTOS), 1995. Directorio. Washington: OMS.

CHETLEY, A., 1994. Qué es un medicamento problema? In: Medicamentos Problema (A. Chetley, ed.), pp 7-32. Amsterdam: HAl.

DA SILVA, C. D. C.; ARRAIS, P. S. D. \& COELHO, H. L. L. CIM/UFC: Um observatório farmacoepidemiológico. III Congresso Brasileiro de Vigilância de Medicamentos. São Paulo, (no prelo).
DA SILVA, C. D. C., 1996. II centro d'informazione sui farmaci dell'Università Federal del Ceará. Resoconto di un anno di attività. Giornale ltaliano di Farmacia Clinica,10:54-57.

DURGLA (DRUG UTILIZATION RESEARCH GROUP/ LATIN AMERICA). Multicentre Study on SelfMedication in Latin-American Countries. Clinical Pharmacology and Therapeutics, (no prelo).

JOSHI, M. D., 1995. New drug information service at a teaching hospital in Nepal. Essential Drugs Monitor, 20:4-5.

LOPES, A. E. C.; TEIXEIRA, A. C. A.; GURGEL, M. L. F.; MIRANDA, M. C. C.; OLIVEIRA, M. A.; OLIVEIRA, M. M. L.; MURTA, R. L. G.; FREITAS, R. M. A.; MENEZES, S. M. M.; BRAGA, W. M. S. \& JONCHERE, K., 1996. Drug use evaluation in health services in Fortaleza, Brazil. INRUD News, 6:17.

MOSDELL, K. W. \& MALONE, P. M., 1996. Drug information resources. In: Drug Information: A Guide for Pharmacists (P. M. Malone, K. W. Mosdell, K. L. Kier \&J. E. Stanovich, eds.), pp. 27-63, Connecticut: Appleton \& Lange. 